\title{
MASS SPECTROMETRIC Fingerprinting OF TANK WASTES USING TUNABLE, ULTRAFAST INFRARED LASERS
}

\author{
RICHARD F. HAGLUND, JR. \\ DEPARTMENT OF PHYSICS AND ASTRONOMY, VANDERBILT UnIVERSITY \\ GRANT NUMBER FG0798ER62710
}

FinAL PROJECT REPORT OCTOBER 1, 1998 - MARCH 31, 2001

Abstract. The principal scientific thrust of this project was to demonstrate a novel method for precision matrix-assisted laser desorption-ionization (MALDI) mass spectrometry (MS) of model tank-waste materials using, using the sodium nitrate component of the tank waste both as the matrix and as an internal calibration standard. Conventional nanosecond and femtosecond, single-frequency lasers and a tunable, mid-infrared free-electron laser were used in the development of the MS protocols and in measurements of the MALDI dynamics. In addition to developing a model of the processes which lead to efficient desorption and ionization of organic molecules (e.g., toluene, benzene, chelators, various organic acids, crown ethers) from sodium nitrate, we developed protocols for quantitative analysis based on the use of the sodium nitrate in tank waste as an internal standard. Comparisons of MALDI-MS using nanosecond and picosecond lasers, and of infrared and ultraviolet lasers, have been especially instructive, and demonstrate the superior potential of IR-MALDI for this purpose, as well as for a number of related analytical and thin-film applications.

Key experimental results. The main thrust of our effort has been to elucidate the mechanisms of MALDI in tank-waste simulants using $\mathrm{NaNO}_{3}$ itself as the matrix, and to demonstrate the potential for quantitative analysis. Most of the experiments involve using a tunable infrared free-electron laser to create ions by ablating the samples at resonant vibrational bands of the matrix. This approach makes it possible to carry out high-precision analysis of the organic content of the simulants without having to add additional matrix material, thus avoiding the possibility of generating unwanted photochemistry during the MS procedure and of creating a potential secondary waste stream. During the project, we have:

- Delineated the roles of laser wavelength, pulse duration, sample $p H$ and mixture complexity in the mass spectra; in particular, we have shown that it is possible to ablate and ionize all the components of a complex tank-waste simulant and to quantitate the results using the characteristic ion peaks of $\mathrm{NaNO}_{3}$ as internal standards.

- Demonstrated that vibrationally excited matrix materials used in analyzing tank waste - such as $\mathrm{NaNO}_{3}$ microcrystallites and 2,5-dihydroxybenzoic acid - are directly implicated in the creation of analyte ions from neutral organic molecules embedded in the matrix, when the laser is tuned to a strong vibrational absorption band.

- Developed a physical model of the processes of desorption, ablation and ionization which can be used both to underpin quantitative analysis and to give insight into the MALDI mechanism in the infrared.

The success of IR-MALDI using $\mathrm{NaNO}_{3}$ as a matrix is surprising (UV-MALDI works best when salts are removed from the matrix-analyte sample), but also points the way to mass spectrometry of organics in other poorly characterized hosts, such as those found associated with the silicate complexes in ordinary dirt. This would make possible, for example, tracking contaminants in the 
vadose zone. We have already initiated collaborative activities along these lines with our colleagues in the Vanderbilt Department of Civil and Environmental Engineering, and expect to submit relevant proposals in the future.

Our experiments and studies of mechanistic models appropriate to IR-MALDI in tankwaste simulants have confirmed the following results:

First, we can indeed detect molecular ions desorbed from $\mathrm{NaNO}_{3}$ with high sensitivity, and there is a linear regime within which one could hope to obtain relatively accurate quantitation. While we are presently only in the low ppm range in terms of sensitivity, we are aware of a number of things we can and will do to enhance the sensitivity by several orders of magnitude. It may be of particular interest that we find the crown ethers, upon desorption, also can abstract and carry away an attached $\mathrm{Na}$ atom which shows up very clearly in the time-of-flight spectrum. [See references 13, 14 below.]

Second, particularly efficient ion production in UV-MALDI is obtained if one irradiates doped $\mathrm{NaNO}_{3}$ at $248 \mathrm{~nm}$ after adding an appropriate matrix material (e.g., dihydroxybenzoic acid), possibly with some penalty in mixtures due to UV-induced photochemistry. On the contrary, however, the addition of infrared MALDI matrices (e.g., succinic acid or 4-hydroxy- $\square$ cyano-cinnamic acid) to the $\mathrm{NaNO}_{3}$ does not materially affect the ion yield, even though the added matrix materials have significant absorption at the $7.1 \square \mathrm{m}$ wavelength used to excite the $\mathrm{NO}_{3}$ stretching vibration. The difference between UV-MALDI and IR-MALDI seems to result from the comparatively greater efficiency for generating photoelectrons in the ultraviolet. [Ref. 17] However, comparison of results across a broad range of wavelengths and pulse durations shows that mass spectra by themselves are not particularly useful in accounting for these mechanistic effects. [Ref. 7]

Third, the "phase explosion" model proposed by a number of researchers seems to describe our results quite nicely — the first time there has been any experimental evidence for this in the infrared. A phase explosion can occur when the optical absorption depth of the material is short compared to the characteristic thermal diffusion length. It is then possible to create very high volumetric density of vibrational excitation at high laser intensity, because of the strong thermal confinement. (In $\mathrm{CaCO}_{3}$, the isoelectronic cousin to $\mathrm{NaNO}_{3}$, the picosecond freeelectron-laser pulses deposit an average of two vibrational quanta in every $\mathrm{CO}_{3}$ group in the irradiated volume.) The irradiated material reaches the critical temperature $\mathrm{T}_{\mathrm{c}}$ for explosive vaporization before the deposited thermal energy has time to equilibrate, leading to the so-called "phase explosion." This should be especially productive of highly energetic ions and neutrals, and gives extremely efficient material removal from surfaces. [Ref. 3]

The major quantitative results for IR-MALDI mixtures in tank simulants will be summarized in a pair of longer papers already prepared for submission in August 2002 [Ref. 13, 14].

Collateral results. The key idea underlying our approach to MALDI-MS in tank-waste simulants is the idea of selective excitation, ablation and ionization based on resonant vibrational, as contrasted with electronic, excitation. This is a novel concept whose experimental realization was dependent upon the available of a tunable, ultrashort-pulse mid-infrared laser capable of exciting specific vibrational modes in many different materials. Our early success in demonstrating the efficacy of this approach to MALDI-MS led us to apply this concept to three important "spin-off" projects that were also investigated during the course of the project.

Desorption of proteins from separation media. Both genomics and proteomics are heavily dependent on mass spectrometry for identification of nucleotides and proteins. In general, MALDI-MS on separation media is a complex and time- 
consuming process, at each step of which the risk of unwanted modification of the analyte is increased. We have shown that resonant IR-MALDI can induce desorption and ionization of proteins directly from a standard polyacrylamide gel. [Refs. 11, 21].

Desorption of proteins from water ice. The native environment for biological molecules is water. However, previous attempts by many investigators to apply IR- and UV-MALDI to the analysis of proteins in water ices have been disappointing. We have been able to reproducibly generate protein ions from water ices both at the $\mathrm{O}-\mathrm{H}$ stretch mode $(2.94 \mu \mathrm{m})$ and $\mathrm{O}-\mathrm{H}$ bending mode $(5.9 \mu \mathrm{m})$ wavelengths. There is reason to believe that the picosecond temporal pulse profile of the free-electron laser plays a major role in the ablation and ionization of the embedded proteins, but that remains as a subject of future investigations. [Refs. 13, 21]

Resonant infrared pulsed laser deposition of polymers. While a few polymers have been successfully deposited as thin films by ultraviolet pulsed laser deposition (PLD), the high probability of photofragmentation prevents this in most cases. Using infrared wavelengths resonant with various vibrational modes of polymers, we have been able to show deposition of intact polymers relevant to electronic, photonic, sensor and medical applications, even for polymers of high molecular weight. [Refs. 5, 8, 14]

Each of these research directions has borne fruit in areas relevant to Department of Energy missions, the first two in the area of proteomics and the last in the development of biological and chemical sensor technology. Two patents have been filed involving these discoveries (described on page 7 of this report), and further proposals and research in these areas are anticipated.

Development of laboratory apparatus. In our proposal, we stated our intent to construct a novel miniature time-of-flight mass spectrometer which would also be suitable for postionization studies of neutral atoms and molecules emitted during laser-induced desorption. This apparatus became fully operational during the summer of 1999, and has provided much of the data needed to test our conjecture about the mechanism of desorption and ionization in the infrared, in particular, the "phase explosion" hypothesis. It was particularly useful in studies of IRMALDI mechanisms using single-ion counting. [Ref. 6]

Training of postdoctoral scholars and graduate students. Substantial technical leadership in our program was provided by Dr. David Ermer (Ph.D., 1997), who was exceptionally adept in developing new hardware and initiating our experiments. He left Vanderbilt for a regular tenuretrack faculty position at Mississippi State University in the fall of 2000. Two graduate students are working on the project: Michael Papantonakis is two or three months away from completing his dissertation; he spent the summer of 1999 working with Drs. Michael Alexander and Wayne Hess in the Environmental Molecular Sciences Laboratory at the Pacific Northwest National Laboratory. His research focus has been the problems of quantitation associated with tank waste and the potential for the use of compact ion-trap mass spectrometers with MALDI ion sources. Michelle Baltz-Knorr is just also nearing the end of her dissertation program, and has overseen the development and fabrication of a cryogenic-target ion source for MALDI using the reflectron time-of-flight spectrometer acquired with research funding from the Office of Naval Research. This has made possible much higher-resolution measurements than we were previously able to carry out, as well as reproducible MALDI-MS using semisolid slurries. Dr. Kenneth Schriver, Research Assistant Professor of Physics, provided much of the day-to-day scientific direction for 
the development of IR-MALDI in water ices during the last months of the project, working with Ms. Baltz-Knorr.

Refereed Publications. The following publications in archival journals and conference proceedings were submitted, accepted or published during this reporting period, all acknowledging Department of Energy sponsorship.

1. "Time-Resolved Studies of Electron-Phonon Relaxation in Metals using a Free-Electron Laser," M. Satta, D. R. Ermer, M. R. Papantonakis, C. Flamini, R. F. Haglund, Jr. and A. Mele, Thin Solid Films 154-155, 172 (2000).

2. "Laser Ablation of Dielectric Materials under Conditions of Strong Vibrational Excitation,” D. R. Ermer, M. R. Papantonakis, M. Baltz-Knorr, D. Nakazawa and R. F. Haglund, Jr., Applied Physics A. 70, 633 (2000).

3. "Explosive vaporization in fused silica initiated by a tunable infrared laser," D. R. Ermer and R. F. Haglund Jr., Applied Surface Science 168, 258-262 (2000).

4. "Pulsed Laser Deposition of Polymer Films Using a Resonantly Tunable Infrared Laser," D. M. Bubb, J. H. Callahan, J. S. Horwitz, R. A. McGill, E. J. Houser, D. B. Chrisey, M. R. Papantonakis, R. F. Haglund, Jr., M. Galicia and A. Vertes, Journal of Vacuum Science and Technology A 19, 2698-2702 (2001). Rapid Communication.

5. "Resonant Infrared Pulsed Laser Deposition of a Sorbent Chemoselective Polymer," D. M. Bubb, D. B. Chrisey, M. R. Papantonakis, R. F. Haglund, Jr., J. S. Horwitz, R. A. McGill and B. Toftmann, Applied Physics Letters 79, 2847-2849 (2001).

6. "Intensity Dependence of Cation Kinetic Energies from 2,5 Dihydroxybenzoic Acid Near IR MALDI Threshold," D. R. Ermer, M. Baltz-Knorr and R. F. Haglund, Jr., Journal of Mass Spectrometry 36 (5), 538-545 (2001).

7. "What do MALDI mass spectra reveal about ionization mechanisms?," J. Kim, W. P. Hess, M. R. Papantonakis and R. F. Haglund, Jr., Journal of Mass Spectrometry 37, 639-647 (2002).

8. "Deposition of thin biodegradable polymer films by resonant infrared pulsed laser deposition,” D. M. Bubb, B. Toftmann, R. F. Haglund, Jr., Jr., J. S. Horwitz, M. R. Papantonakis, R. A. McGill, P. W. Wu, and D. B. Chrisey, Applied Physics A 121, 123-125 (2002).

9. "Vapor Deposition of Polystyrene Thin Films by Intense Laser Excitation of Resonant Vibrational Modes," D. M. Bubb, M. R. Papantonakis, R. F. Haglund, Jr., J. S. Horwitz, R. A. McGill and D. B. Chrisey, Chemical Physics Letters 352, 135-139 (2002).

10. "Infrared Laser Desorption and Ionization of Polypeptides from a Polyacrylamide Gel," M. L. Baltz-Knorr, D. R. Ermer, K. E. Schriver and R. F. Haglund, Jr., accepted for publication, Journal of Mass Spectrometry 37, 254-258 (2002). Accelerated Communication.

11. "The effect of ablation parameters on infrared pulsed laser deposition of poly(ethylene glycol) thin films," Daniel M. Bubb, M. R. Papantonakis, B. Toftmann, J. S. Horwitz, R. A. McGill, D. B. Chrisey and R. F. Haglund, Jr., Journal of Applied Physics 91, 9809-9814 (2002).

12. "Infrared MALDI mass spectrometry of carboxylic acids from sodium nitrate," M. R. Papantonakis and R. F. Haglund, Jr., manuscript ready for submission to Analytical and Bioanalytical Chemistry, August 2002. 
13. "Sensitive mass spectrometric probing of complex mixtures using a tunable, picosecond infrared laser," R. F. Haglund, Jr., M. Baltz-Knorr, D. R. Ermer, M. R. Papantonakis and K. E. Schriver, manuscript submitted to Spectrochimica Acta, August 2002. Invited review paper.

14. "Deposition of thin poly(tetrafluoroethylene) films at room temperature by resonant pulsed laser evaporation," M. R. Papantonakis, D. M. Bubb, J. S. Horwitz and R. F. Haglund, Jr., manuscript in preparation for submission to Applied Physics Letters, September 2002.

15. "First observation of a matrix suppression effect in infrared matrix-assisted laser desorption and ionization of small peptides," M. R. Papantonakis and R. F. Haglund, Jr., manuscript in preparation for submission to Journal of Mass Spectrometry, September 2002.

Conference proceedings. The following publications in conference proceedings were submitted, accepted or published during this reporting period, all acknowledging Department of Energy sponsorship. Those articles that were peer reviewed are marked with a *.

16. "Vibrational excitation and relaxation processes in insulators initiated by ultrashort, midinfrared laser pulses," R. F. Haglund Jr., R. Cramer, D. R. Ermer, M. R. Papantonakis, H. K. Park and O. Yavas' in Laser Applications in Microelectronic and Optoelectronic Manufacturing IV, eds. J. J. Dubowski, H. Helvajian, E. W. Kreutz, K. Sugioka, SPIE 3618, 90101 (1999).

17. *"Matrix-assisted infrared-laser desorption-ionization mass spectrometry of organic molecules on $\mathrm{NaNO}_{3}$," D. R. Ermer, M. Baltz-Knorr, D. Nakazawa, M. R. Papantonakis, and R. F. Haglund, Jr. in Scientific Basis for Nuclear Waste Management XXIII, eds. R. W. Smith and D. W. Shoesmith, Mat. Res. Soc. Symp. Proc. 608, 613-618 (2000).

18. "Charged-particle emission from dielectric materials initiated by a tunable, picosecond mid-infrared laser,” D. R. Ermer, M. R. Papantonakis, M. Baltz-Knorr and R. F. Haglund, Jr., Proc. SPIE 3935, 104-112 (2000).

19. "Phase Explosion and Ablation Initiated in Fused Silica Initiated by a Tunable, UltrashortPulse Mid-Infrared Laser," R. F. Haglund, Jr., D. R. Ermer, H. K. Park, M. R. Papantonakis and O. Yavas, Proc. SPIE 4065, 132-143 (2001).

20. *Infrared Laser Ablation and Ionization of Water Clusters and Biomolecules from Ice," M. L. Baltz-Knorr, K. E. Schriver and R. F. Haglund, Jr., in press, Applied Surface Science (2002).

21. *"Picosecond Infrared Matrix-Assisted Desorption and Ionization of Organic Molecules in Nitrate Crystals," M. R. Papantonakis, D. R. Ermer and R. F. Haglund, Jr., in press, Applied Surface Science (2002)

22. "Deposition of chemically sensitive polymer films by picosecond resonant infrared laser ablation," D. M. Bubb, J. A. Callahan, R. F. Haglund, Jr., J. S. Horwitz, E. A. Houser, R. A. McGill and M. R. Papantonakis, in High-Power Laser Ablation 2002, ed. C. R. Phipps, Jr., Proc. SPIE 4760, in press (2002)

Conference contributions. A number of conference contributions and publications were presented as a result of this year's work, including papers at the annual meeting of the American Society for Mass Spectrometry and a number of invited talks. The following talks acknowledged DOE sponsorship (the name of the presenting author is italicized): 
Plenary lecture, "The Future of Tunable, Ultrafast Lasers in Materials Analysis and Processing," R. F. Haglund, Jr., American Society for Mass Spectrometry, Dallas, TX, June 1999.

Invited talk, "The Role of Vibrational Excitation in Desorption and Ionization from Molecular Solids," R. F. Haglund, Jr., Conference on Laser Ablation (COLA'99), Goettingen, Germany, July 1999.

"Measuring Molecules by the Attomole," R. F. Haglund, Jr., Southeastern Section Meeting of the American Physical Society, Chapel Hill, NC, November 7-9, 1999. Invited talk.

"Phase Explosions in Solid Surfaces Initiated by Ultrafast Laser Irradiation in the Mid-Infrared," R. F. Haglund, Jr., SPIE High Power Laser Applications Conference (HPLA-III), Santa Fe, NM, April 23-28, 2000. Invited talk.

"IR-MALDI Response from Various Absorption Modes," M. R. Papantonakis, $48^{\text {th }}$ Annual Meeting of the American Society for Mass Spectrometry, San Diego, CA, June 1-4,2000.

"Intensities and energy distributions of charged particles emitted from positional isomorphs of dihydroxybenoic acid during irradiation at $2.9 \square \mathrm{m}$," D. R. Ermer, $48^{\text {th }}$ Annual Meeting of the American Society for Mass Spectrometry, San Diego, CA, June 1-4,2000.

"Rethinking laser ablation: mechanisms of excitation and relaxation at the picosecond time scale," R. F. Haglund, Jr., Gordon Research Conference on "Laser Interactions with Materials," Proctor Academy, June 16-20, 2000. Invited talk.

"Mechanism of Infrared-Laser Desorption and Ionization of Molecules Embe dded in Solid and Liquid Matrices," M. L. Baltz-Knorr, D. R. Ermer and R. F. Haglund, Jr. and "Micromachining of Glasses by Explosive Vaporization using a Tunable, Mid-Infrared Free-Electron Laser," ;D. R. Ermer, M. R. Papantonakis and R. F. Haglund, Jr., contributed papers presented at the International FreeElectron Laser Conference FEL-2000, Duke University, August 2000.

"Molecular identification of mixed tank waste compounds by mass spectrometry," M. R. Papantonakis, American Chemical Society Meeting, EMSP Session, Chicago, IL, August 24-27, 2000.

"Desorption and Ionization of Peptides and Proteins using a Tunable, MidInfrared Laser," R. F. Haglund, Jr., M. L. Baltz-Knorr, D. R. Ermer and M. R. Papantonakis, contributed paper at "invitation only" meeting, Desorption 2000 Conference, St. Malo, France, September 7-10, 2000.

"Mass spectrometry of proteins using an infrared free-electron laser," M. BaltzKnorr, D. R. Ermer, M. R. Papantonakis and R. F. Haglund, Jr., contributed paper, American Physical Society Southeastern Section Meeting, November 2000.

"Biological Mass Spectrometry using a Picosecond Mid-Infrared Free-Electron Laser," Michelle Baltz-Knorr, D. R. Ermer, M. R. Papantonakis and R. F. Haglund, Jr., invited presentation at the meeting of the Photon Consortium, Jefferson National Accelerator Laboratory, Newport News, VA, January 18, 2001.

"The convergence of materials and biological physics at mesoscopic length scales," R. F. Haglund, Jr., L. C. Feldman, S. Rosenthal, R. A. Weller and J. P. 
Wikswo, invited talk, American Physical Society March Meeting, Seattle, WA, March 16-20, 2001.

R. F. Haglund, Jr., "Processing and Analysis of Materials using Tunable, Ultrashort-pulse Lasers in the Mid-Infrared," Materials Research Society Spring Meeting, San Francisco, CA, April 2001. Invited talk.

"IR-MALDI Mass Spectrometry of Polyacrylamide Gels and Water/Ice at MidInfrared Wavelengths," Michelle Baltz-Knorr, David R. Ermer, Michael R. Papantonakis, Kenneth E. Schriver, Richard F. Haglund, Jr. "Mass Spectrometric Detection of Complex Organic Mixtures in Simulated Nuclear Tank Wastes," $M$. R. Papantonakis, D. R. Ermer, W. P. Hess and R. F. Haglund, Jr., American Society of Mass Spectrometry, 49 ${ }^{\text {th }}$ Annual Meeting, Chicago, May 2001.

"Pulsed laser deposition of polymer films by resonant vibrational ablation," R. F. Haglund, Jr., M. R. Papantonakis, D. M. Bubb, J. A. Horwitz, Symposium L, European Materials Research Society Meeting, Strasbourg, June 19-22, 2001.

R. F. Haglund, Jr., "Free-Electron-Laser Based Mass Spectrometry for Biological and Environmental Applications," International Free-Electron Laser Conference FEL-2001, Darmstadt, Germany, August 20-24, 2001. Invited talk.

"Infrared Matrix-Assisted Laser Desorption-Ionization Mass Spectrometry for Environmental Applications using Tunable Ultrafast Laser Pulses," $M . R$. Papantonakis, D. R. Ermer and R. F. Haglund, Jr., American Society for Mass Spectrometry Workshop on Environmental Mass Spectrometry, Asilomar, CA, October 2001.

"Infrared Laser Ablation and Ionization of Water Clusters and Biomolecules from Ice," M. Baltz-Knorr, K. E. Schriver and R. F. Haglund, Jr.; "Picosecond Infrared Matrix-Assisted Desorption and Ionization of Organic Molecules in Nitrate Crystals," M. R. Papantonakis, D. R. Ermer and R. F. Haglund, Jr., presented at the International Conference on Laser Ablation (COLA-VI), Tsukuba, Japan, October 2001.

Patent applications. We filed two applications for patents relating to this research program during the course of the past three years.

"Method and technology for pulsed laser evaporation of thin polymer films using resonant infrared laser ablation," provisional patent application filed jointly by the Naval Research Laboratory and Vanderbilt University; inventors were Daniel M. Bubb, John H. Callahan, Richard F. Haglund, Jr., James S. Horwitz and Michael R. Papantonakis, July 2001.

"Method and Apparatus for the Mass Spectrometry of Proteins and Nucleotides Embedded in Electrophoresis Gels," international patent application filed August 2001 at Vanderbilt University on behalf of David R. Ermer, Michelle L. Baltz-Knorr and Richard F. Haglund, Jr.. 